\title{
On the motion of billiards in ellipses
}

\author{
Hellmuth Stachel ${ }^{1}$ (D)
}

Received: 12 May 2021 / Revised: 28 September 2021 / Accepted: 7 December 2021 /

Published online: 24 January 2022

(c) The Author(s) 2022

\begin{abstract}
For billiards in an ellipse $e$ with an ellipse as caustic, there exist canonical coordinates on $e$ such that the billiard transformation from vertex to vertex is equivalent to a shift of coordinates. A kinematic analysis of billiard motions offers a new approach to canonical parametrizations of billiards and associated Poncelet grids. This parametrization uses Jacobian elliptic functions with the modulus equal to the numerical eccentricity of the caustic and is the basis for proving a few invariants of periodic billiards.
\end{abstract}

Keywords Billiard · Billiard motion · Confocal conics · Elliptic functions

Mathematics Subject Classification 51N20 $\cdot$ 53A17 $\cdot 33 \mathrm{E} 05 \cdot 22 \mathrm{E} 30$

\section{Introduction}

A billiard is the trajectory of a mass point within a domain with ideal physical reflections in the boundary, which sometimes is called a billiard table. Already for two centuries, billiards in ellipses and their projectively equivalent counterparts have attracted the attention of mathematicians, beginning with Jean-Victor Poncelet and Carl Gustav Jacob Jacobi. The assertion that one $N$-periodic billiard inscribed in an ellipse $e$ and tangent to a confocal ellipse $c$ called caustic implies a one-parameter family of such polygons, is known as the standard example of a Poncelet porism. It was Arthur Cayley who derived general algebraic conditions for the existence of an $N$-sided polygon with a circumscribed conic $e$ and an inscribed conic $c$.

In 2005, Tabachnikov published the book on billiards [21] which covers a wide variety of themes around this topic. Dragović and Radnović addressed billiards in conics and quadrics, even in higher dimensions, within the framework of dynamical systems (see the book [6] and various papers, e.g., [5,7]).

\footnotetext{
Hellmuth Stachel

stachel@dmg.tuwien.ac.at

1 Institute of Discrete Mathematics and Geometry, Vienna University of Technology, Vienna, Austria
} 
Recent computer animations carried out by Reznik, stimulated a new vivid interest on this well studied topic, where algebraic and analytic methods are meeting. Originally, Reznik's experiments focused on billiard motions in ellipses, i.e., on the variation of billiards with a fixed circumscribed ellipse $e$ and inscribed caustic $c$. For periodic billiards he published a list of more than 80 numerically detected invariants in [17] and provided several proofs together with his coauthors Garcia, Koiller and Helman. Also other authors like Akopyan, Bialy, Chavez-Caliz, Schwartz, and Tabachnikov published proofs and found additional invariants (e.g., in $[1,2,4])$.

Related work. Since Jacobi's proof of the Poncelet theorem on closed polygons with a circumcircle and incircle in 1828 [14], it has been well known that there is a tight connection between billiards and elliptic functions (see further references in [5, p. 320] and note also [8]). These functions enable to parametrize the circumscribed conic such that the billiard transformation from one vertex to the next one acts like a shift. We call such parameters canonical. In 1994, King proved the existence of a canonical parameter using measure theory; he presented an explicit formula in [15, (1.10)]. In [21], Tabachnikov showed the existence of canonical parameters with the ArnoldLiouville theorem from the theory of completely integrable systems (see also [13]).

Similarly, the caustic is said to have Poritsky property if there is a parametrization such that the transition from one contact point of the billiard to the next one is a shift. This is due to Poritsky [16] who proved in 1950, that this property characterizes ellipses as boundary. In [16, (8.1)] he presented a formula for this parameter, which we call the Poritzky string length. Glutsyuk, Izmestiev and Tabachnikov generalized Poritzky's result in [11] and proved equivalences between the Poritsky property, the Graves property and the Ivory property of curves on Riemannian surfaces. Glutsyuk presented in [10] an expression for the Poritsky string length for caustics which is valid on all Riemannian surfaces of constant curvature.

Article structure. The main goal of this paper is to demonstrate that a velocity analysis of the billiard motion paves the way to an explicit canonical representation of the said billiards in terms of Jacobian elliptic functions.

In Sect. 2 we present a summary of relevant properties of confocal conics. A graphical velocity analysis of the billiard motion and its continuation to the associated Poncelet grid in Sect. 3 results in a new motion invariant. This is the basis for a new approach to canonical coordinates on the ellipse $e$ as presented in Sect. 4: The velocity vectors define an infinitesimal transformation in the plane which preserves a family of confocal ellipses while it permutes the confocal hyperbolas as well as the tangents of the caustic.

The infinitesimal transformation generates a one-parameter Lie group. Its canonical parametrization uses Jacobian elliptic functions with the numerical eccentricity of the caustic $c$ as modulus and yields canonical coordinates on each ellipse of the confocal family and simultaneously a Poritzky string length on the caustic. Moreover, this parametrization gives rise to a mapping that sends a square grid together with the diagonals to a Poncelet grid. It is an extension of a mapping shown in [7, Figure 13], which transforms a zig-zag polygon into a billiard.

Finally, in Sect. 5 the foregoing results are used to prove for periodic billiards the numerically detected invariants k116 and k117 out of Reznik's list [17] and to reveal 
two new invariants in the Theorems 5.2 and 5.4. These invariants deal mainly with the distances on each billiards' side between the endpoint and the contact point with the caustic.

In the present paper, we confine ourselves on billiards in ellipses with an ellipse as caustic. Similar results for cases with a hyperbola as caustic can be directly deduced using the isometric correspondence between the two types as presented in [19, Theorem 1].

\section{Confocal conics and billiards}

At the beginning, we recall a few properties of confocal conics. A family of confocal central conics is given by

$$
\frac{x^{2}}{a^{2}+k}+\frac{y^{2}}{b^{2}+k}=1, \quad \text { where } k \in \mathbb{R} \backslash\left\{-a^{2},-b^{2}\right\}
$$

serves as a parameter in the family. All these conics share the focal points $F_{1,2}=$ $( \pm d, 0)$, where $d^{2}:=a^{2}-b^{2}$.

Given any confocal family of central conics, there is one ellipse and one hyperbola containing any point $P$ which is not placed on the axes [9, p.38]. The parameters $\left(k_{e}, k_{h}\right)$ of these two conics define the elliptic coordinates of $P$ with

$$
-a^{2}<k_{h}<-b^{2}<k_{e}
$$

If $(x, y)$ are the Cartesian coordinates of $P$, then $\left(k_{e}, k_{h}\right)$ are the roots of the quadratic equation

$$
k^{2}+\left(a^{2}+b^{2}-x^{2}-y^{2}\right) k+\left(a^{2} b^{2}-b^{2} x^{2}-a^{2} y^{2}\right)=0,
$$

while conversely

$$
x^{2}=\frac{\left(a^{2}+k_{e}\right)\left(a^{2}+k_{h}\right)}{d^{2}}, \quad y^{2}=-\frac{\left(b^{2}+k_{e}\right)\left(b^{2}+k_{h}\right)}{d^{2}} .
$$

Suppose that $(a, b)$ in (2.1) are the semiaxes $\left(a_{c}, b_{c}\right)$ of the ellipse $c$ with $k=0$. Then, for points $P$ on a confocal ellipse $e$ with semiaxes $\left(a_{e}, b_{e}\right)$ and $k=k_{e}>0$, i.e., exterior to $c$, the standard parametrization yields

$$
P=(x, y)=\left(a_{e} \cos t, b_{e} \sin t\right), \quad 0 \leqslant t<2 \pi,
$$

with $a_{e}^{2}=a_{c}^{2}+k_{e}, b_{e}^{2}=b_{c}^{2}+k_{e}$. For the elliptic coordinates $\left(k_{e}, k_{h}\right)$ of $P$ it follows from (2.2) that

$$
k_{e}+k_{h}=a_{e}^{2} \cos ^{2} t+b_{e}^{2} \sin ^{2} t-a_{c}^{2}-b_{c}^{2} .
$$




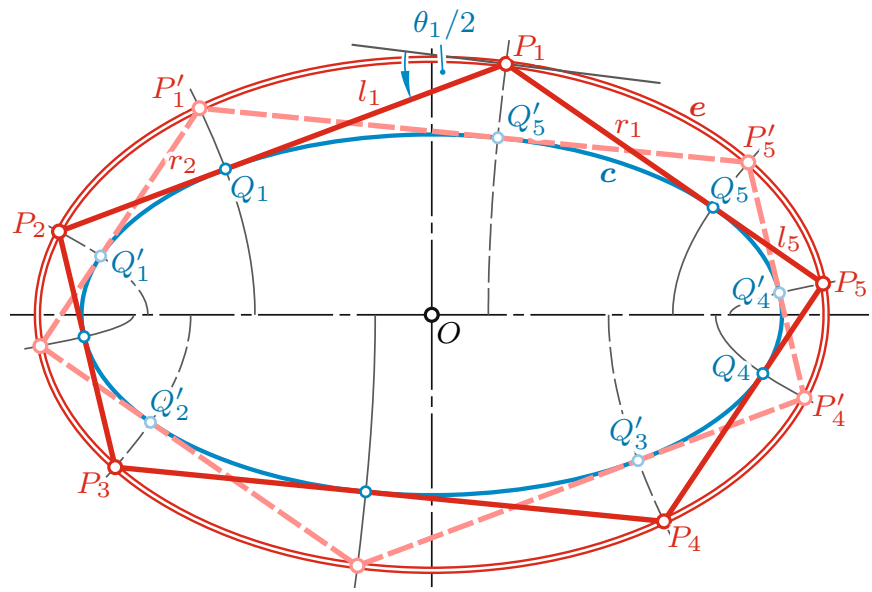

Fig. 1 The periodic billiard $P_{1} P_{2} \ldots P_{5}$ inscribed in $e$ with the caustic $c$ along with the conjugate billiard $P_{1}^{\prime} P_{2}^{\prime} \ldots P_{5}^{\prime}$

After introducing the respective tangent vectors of $e$ and $c$, namely

$$
\mathbf{t}_{e}(t):=\left(-a_{e} \sin t, b_{e} \cos t\right), \quad \mathbf{t}_{c}(t):=\left(-a_{c} \sin t, b_{c} \cos t\right),
$$

where $\left\|\mathbf{t}_{e}\right\|^{2}=\left\|\mathbf{t}_{c}\right\|^{2}+k_{e}$, we obtain

$$
k_{h}=k_{h}(t)=-\left(a_{c}^{2} \sin ^{2} t+b_{c}^{2} \cos ^{2} t\right)=-\left\|\mathbf{t}_{c}(t)\right\|^{2}=-\left\|\mathbf{t}_{e}(t)\right\|^{2}+k_{e}
$$

and $\left\|\mathbf{t}_{e}(t)\right\|^{2}=k_{e}-k_{h}(t)$. Note that points on the confocal ellipses $e$ and $c$ with the same parameter $t$ have the same coordinate $k_{h}$. Consequently, they belong to the same confocal hyperbola (Fig. 2). Conversely, points of $e$ or $c$ on this hyperbola have a parameter out of $\{t,-t, \pi+t, \pi-t\}$ modulo $2 \pi$.

Let $\theta_{i} / 2$ denote the angle between the tangents drawn from any point $P_{i} \in e$ to $c$ and the tangent to $e$ at $P_{i}$ (Figs. 1 or 3). Then we obtain for $P_{i}=\left(a_{e} \cos t_{i}, b_{e} \sin t_{i}\right)$ with elliptic coordinates $\left(k_{e}, k_{h}\left(t_{i}\right)\right)$

$$
\begin{aligned}
& \sin ^{2} \frac{\theta_{i}}{2}=\frac{k_{e}}{\left\|\mathbf{t}_{e}\left(t_{i}\right)\right\|^{2}}=\frac{k_{e}}{k_{e}-k_{h}\left(t_{i}\right)}, \quad \tan \frac{\theta_{i}}{2}= \pm \sqrt{-\frac{k_{e}}{k_{h}\left(t_{i}\right)}} \\
& \text { and } \sin \theta_{i}= \pm \frac{2 \sqrt{-k_{e} k_{h}\left(t_{i}\right)}}{k_{e}-k_{h}\left(t_{i}\right)}= \pm \frac{2\left\|\mathbf{t}_{c}\left(t_{i}\right)\right\| \sqrt{k_{e}}}{\left\|\mathbf{t}_{e}\left(t_{i}\right)\right\|^{2}}
\end{aligned}
$$

For a proof see [20]. We assume a counter-clockwise order of the billiard. Hence, all exterior angles $\theta_{i}$ are positive.

From (2.4) it follows that

$$
k_{h}=-\frac{a_{c}^{2} \tan ^{2} t+b_{c}^{2}}{1+\tan ^{2} t}, \text { hence } \tan ^{2} t\left(a_{c}^{2}+k_{h}\right)=-b_{c}^{2}-k_{h}
$$




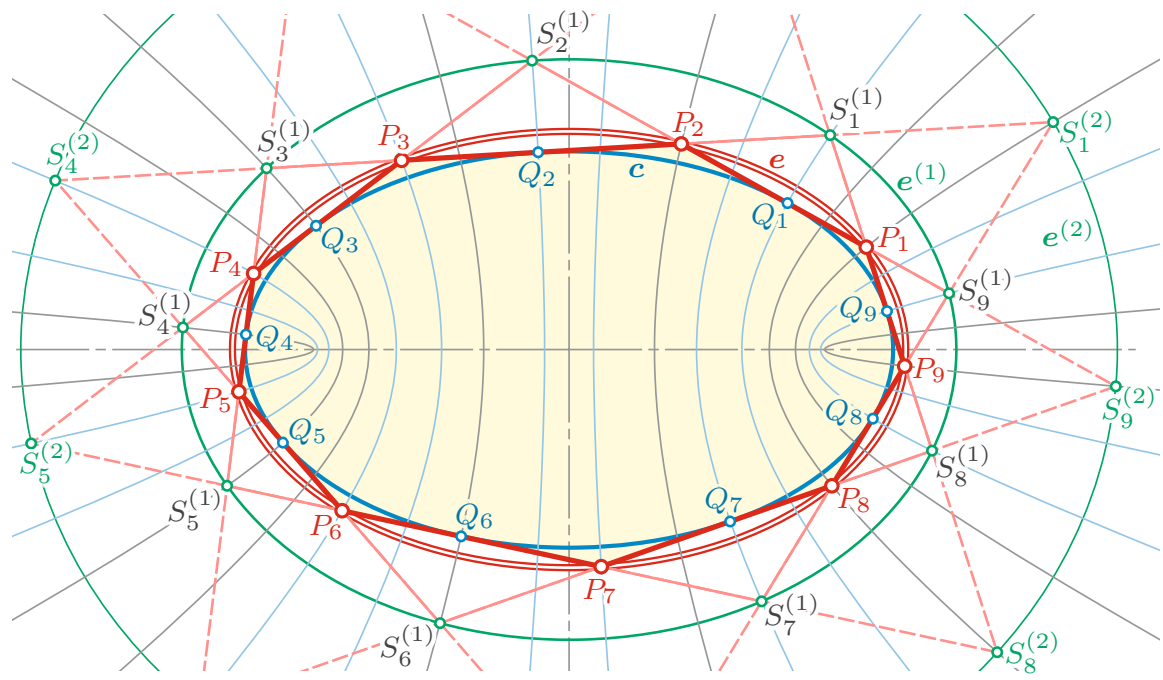

Fig. 2 Periodic billiard $P_{1} P_{2} \ldots P_{9}$ in $e$ with turning number $\tau=1$, the caustic $c$ and a part of the associated Poncelet grid. The extended sides form a billiard with $\tau=2$ in $e^{(1)}$ and three triangles as billiards in $e^{(2)}$

and furthermore

$$
\sin t \cos t=\frac{\tan t}{1+\tan ^{2} t}=\frac{\sqrt{-\left(b_{c}^{2}+k_{h}\right)\left(a_{c}^{2}+k_{h}\right)}}{a_{c}^{2}-b_{c}^{2}}=\frac{a_{h} b_{h}}{d^{2}}
$$

with $a_{h}$ and $b_{h}$ as semiaxes of the hyperbola corresponding to the parameter $t$, i.e., $a_{h}^{2}=a_{c}^{2}+k_{h}$ and $b_{h}^{2}=-\left(b_{c}^{2}+k_{h}\right)$.

Let ... $P_{1} P_{2} P_{3} \ldots$ be a billiard in the ellipse $e$ with the confocal ellipse $c$ as caustic. Then the extended sides intersect at points

$$
S_{i}^{(j)}:=\left\{\begin{array}{l}
{\left[P_{i-k-1}, P_{i-k}\right] \cap\left[P_{i+k}, P_{i+k+1}\right] \text { for } j=2 k,} \\
{\left[P_{i-k}, P_{i-k+1}\right] \cap\left[P_{i+k}, P_{i+k+1}\right] \text { for } j=2 k-1,}
\end{array}\right.
$$

where $i=\ldots, 1,2,3, \ldots$ and $j=1,2, \ldots$ These points are distributed on different confocal conics: For fixed $j$, there are ellipses $e^{(j)}$ passing through the points $S_{i}^{(j)}$. On the other hand, the points $S_{i}^{(2)}, S_{i}^{(4)}, \ldots$ are located on the confocal hyperbola through $P_{i}$, while $S_{i}^{(1)}, S_{i}^{(3)}, \ldots$ belong to the confocal hyperbola through the contact point $Q_{i}$ between the side $P_{i} P_{i+1}$ and the caustic $c$. This configuration is called the associated Poncelet grid (Fig. 2). For periodic billiards the sets of points $S_{i}^{(j)}$ and associated conics are finite. The turning number $\tau$ of a periodic billiard in $e$ with an ellipse as caustic counts how often one period of the billiard surrounds the center $O$ of $e$ (note Fig. 2).

For each billiard $P_{1} P_{2} \ldots$ in $e$ with caustic $c$ there exists a conjugate billiard $P_{1}^{\prime} P_{2}^{\prime} \ldots$ in $e$ with the same caustic (Fig. 1). An axial scaling $c \rightarrow e$ maps the contact point $Q_{i} \in c$ of $P_{i} P_{i+1}$ to $P_{i}^{\prime}$ while the inverse brings $P_{i}$ to the contact point $Q_{i-1}^{\prime}$ of 


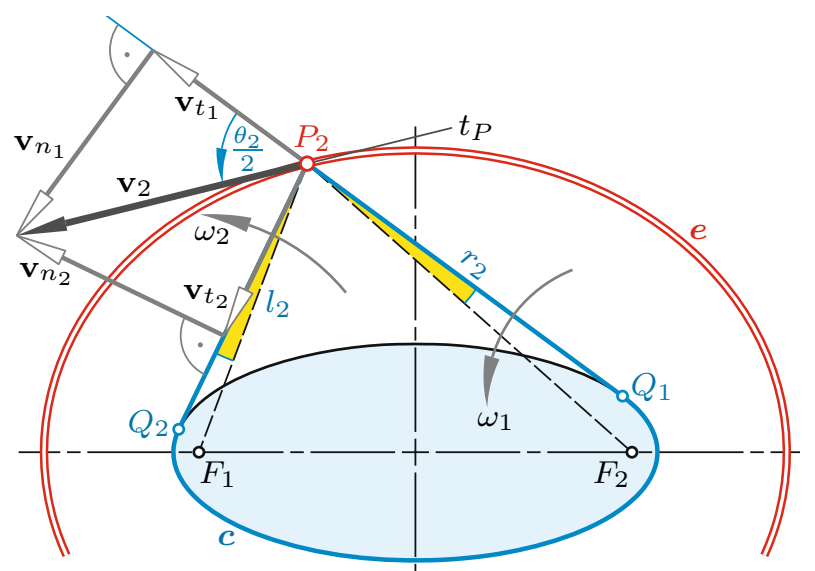

Fig. 3 Velocity analysis resulting from Graves' string construction of the ellipse $e$

$P_{i-1}^{\prime} P_{i}^{\prime}$ with the caustic. The relation between these billiards is symmetric and shows that any canonical parameter of $e$ serves also as Poritzky string length on $c$. In the sense of canonical coordinates, the vertices $P_{1}^{\prime} P_{2}^{\prime} \ldots$ are the midpoints between consecutive vertices of the original billiard. For further details on the associated Poncelet grid see [20, Section 3.2] and the references cited there.

\section{Velocity analysis}

Let the first vertex of a billiard $P_{1} P_{2} \ldots$ move smoothly along the circumscribed ellipse $e$. Then this induces a continuous variation of all other vertices along $e$ and also of the intersection points $S_{i}^{(j)}$ along $e^{(j)}$ (see [1] or [20]). We call this a billiard motion, though it neither preserves angles or distances nor is an affine or projective motion .

According to Graves' construction [9, p. 47], we can assume that each vertex $P_{i} \in e$ keeps a string of fixed length taut which is wrapped around the caustic $c$ without slipping on $c$. We translate this in the language of kinematics.

Let us focus on the vertex $P_{2}$ (see Fig. 3). The extended line $\left[Q_{1}, P_{2}\right]$ of the side $Q_{1} P_{2}$ rolls at $Q_{1}$ on $c$ (=fixed polode) while point $P_{2}$ moves along this line (= moving polode) with the velocity vector $\mathbf{v}_{t_{1}}$. The instantaneous rotation about $Q_{1}$ with the angular velocity $\omega_{1}$ assigns to $P_{2}$ a velocity vector $\mathbf{v}_{n_{1}}$ orthogonal to $Q_{1} P_{2}$ in order to keep the vector of absolute velocity of $P_{2}$, namely $\mathbf{v}_{2}=\mathbf{v}_{t_{1}}+\mathbf{v}_{n_{1}}$, tangent to the ellipse $e$.

Similarly, we have a second decomposition $\mathbf{v}_{2}=\mathbf{v}_{t_{2}}+\mathbf{v}_{n_{2}}$, since at the same time the line $\left[Q_{2}, P_{2}\right]$ rotates about $Q_{2}$ with the angular velocity $\omega_{2}$, while $P_{2}$ moves relative to this line. Due to the constant length of the string, the tangential components in these two decompositions must be of equal lengths $\left\|\mathbf{v}_{t_{2}}\right\|=\left\|\mathbf{v}_{t_{1}}\right\|$. Since the tangent $t_{P}$ to $e$ at $P_{2}$ bisects the exterior angle of $Q_{1} P_{2} Q_{2}$, the second decomposition is symmetric with respect to (w.r.t. in brief) $t_{P}$ to the first one. From $\left\|\mathbf{v}_{n_{2}}\right\|=\left\|\mathbf{v}_{n_{1}}\right\|$ it follows for 


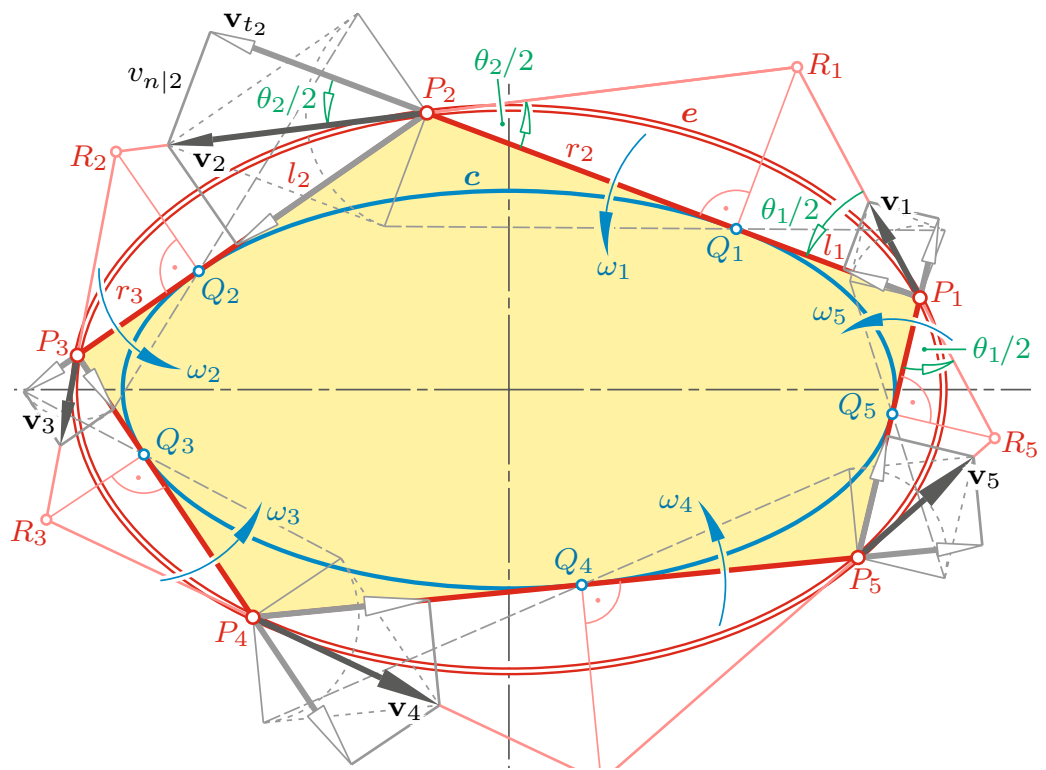

Fig. 4 Velocities of the vertices $P_{1}, P_{2}, \ldots, P_{5}$ of a periodic billiard in the ellipse $e$ with the caustic $c$

the distances $r_{2}:=\overline{P_{2} Q_{1}}$ and $l_{2}:=\overline{P_{2} Q_{2}}$ that

$$
l_{2} \omega_{2}=r_{2} \omega_{1}, \quad \text { or } \frac{\omega_{1}}{\omega_{2}}=\frac{l_{2}}{r_{2}},
$$

and similarly for all other vertices. If the billiard is $N$-periodic, then the product of all ratios $l_{i} / r_{i}$ for $i=1, \ldots, N$ yields

$$
\frac{l_{1}}{r_{1}} \cdot \frac{l_{2}}{r_{2}} \cdots \frac{l_{N}}{r_{N}}=\frac{\omega_{N}}{\omega_{1}} \cdot \frac{\omega_{1}}{\omega_{2}} \cdots \frac{\omega_{N-1}}{\omega_{N}}=1,
$$

which results in the equation

$$
l_{1} l_{2} \ldots l_{N}=r_{1} r_{2} \ldots r_{N}
$$

listed as k116 in [17, Table 2].

Figure 4 shows a graphical velocity analysis for the billiard motion of a 5-sided periodic billiard. We can begin this analysis by choosing an arbitrary length for the arrow representing the velocity vector $\mathbf{v}_{2}$ of $P_{2}$. This defines the two components $\mathbf{v}_{t_{2}}$ and $\mathbf{v}_{n_{2}}$, where the latter determines the angular velocity $\omega_{2}$ of the side $P_{2} P_{3}$ and furtheron the absolute velocity $\mathbf{v}_{3}$ of $P_{3}$. This can be continued. From now on, we denote the norms $\left\|\mathbf{v}_{t_{1}}\right\|=\left\|\mathbf{v}_{t_{2}}\right\|$ and $\left\|\mathbf{v}_{n_{1}}\right\|=\left\|\mathbf{v}_{n_{2}}\right\|$ of the respective components of the velocity vector $\mathbf{v}_{i}$ of $P_{i}$ with $v_{t \mid i}$ and $v_{n \mid i}$. 
In terms of the exterior angles $\theta_{1}, \theta_{2}, \ldots$ of the billiard, we obtain from (3.1)

$$
\sin \frac{\theta_{2}}{2}=\frac{l_{2} \omega_{2}}{v_{2}}=\frac{r_{2} \omega_{1}}{v_{2}} \text { and } \cos \frac{\theta_{2}}{2}=\frac{v_{t \mid 2}}{v_{2}}, \quad \text { where } v_{2}:=\left\|\mathbf{v}_{2}\right\|
$$

Let $R_{i}$ denote the pole of the line $\left[P_{i}, P_{i+1}\right]$ w.r.t. $e$. Since the poles of a line $\ell$ w.r.t. confocal conics lie on a line orthogonal to $\ell$, the side $P_{1} P_{2}$ is orthogonal to $\left[Q_{1}, R_{1}\right]$ (Fig. 4), which means

$$
\overline{R_{1} Q_{1}}=l_{1} \tan \frac{\theta_{1}}{2}=r_{2} \tan \frac{\theta_{2}}{2}
$$

From (3.4) and (3.3) it follows that

$$
l_{1} \tan \frac{\theta_{1}}{2}=l_{1} \frac{l_{1} \omega_{1}}{v_{t \mid 1}}=r_{2} \tan \frac{\theta_{1}}{2}=r_{2} \frac{r_{2} \omega_{1}}{v_{t \mid 2}} \text { and } \frac{v_{t \mid 2}}{v_{t \mid 1}}=\frac{r_{2}^{2}}{l_{1}^{2}}=\frac{\tan ^{2}\left(\theta_{1} / 2\right)}{\tan ^{2}\left(\theta_{2} / 2\right)}
$$

This shows by virtue of (2.5), that the products

$$
v_{t \mid 1} \tan ^{2} \frac{\theta_{1}}{2}=v_{t \mid 2} \tan ^{2} \frac{\theta_{2}}{2}=\cdots=v_{t \mid i} \frac{k_{e}}{\left\|\mathbf{t}_{c \mid i}\right\|^{2}}
$$

for $i=1,2, \ldots$ remain constant along the billiard. We denote this quantity temporarily with $C$ and recognize that this is a new invariant of the billiard motion.

Instead of a free choice of $v_{2}$, it means no restriction of generality to set $C=k_{e}$. Then we obtain by (2.5) for the point $P_{i}=\left(a_{e} \cos t_{i}, b_{e} \sin t_{i}\right)$ of the ellipse $e$,

$$
\begin{aligned}
v_{t \mid i} & =\left\|\mathbf{t}_{c}\right\|^{2}=-k_{h}, \quad v_{n \mid i}=v_{i} \sin \frac{\theta_{i}}{2}=\left\|\mathbf{t}_{c}\right\| \sqrt{k_{e}}=\sqrt{-k_{e} k_{h}}, \\
v_{i} & =\frac{\left\|\mathbf{t}_{c}\right\|^{2}}{\cos \left(\theta_{i} / 2\right)}=\left\|\mathbf{t}_{c}\right\|\left\|\mathbf{t}_{e}\right\|=\sqrt{k_{h}\left(k_{h}-k_{e}\right)} \text { for } t=t_{i} \text { and } k_{h}=k_{h}\left(t_{i}\right) .
\end{aligned}
$$

\section{Billiard motion and the underlying Lie group}

Our specification of the quantity $C$ assigns to the vertex $P_{i} \in e$ with parameter $t_{i}$ a non-vanishing velocity vector $\mathbf{v}_{i}=\left\|\mathbf{t}_{c}\left(t_{i}\right)\right\| \mathbf{t}_{e}\left(t_{i}\right)$. This assignment can immediately be extended to all points of $e$ as

$$
\mathbf{v}(t)=\left\|\mathbf{t}_{c}(t)\right\| \mathbf{t}_{e}(t)=\sqrt{a_{c}^{2} \sin ^{2} t+b_{c}^{2} \cos ^{2} t} \mathbf{t}_{e}(t) .
$$

There exists a parameter $u$ on $e$ such that the differentiation by $u$ results in the said velocity vector. If a dot indicates this differentiation, then

$$
\mathbf{v}(t)=\left\|\mathbf{t}_{c}(t)\right\| \mathbf{t}_{e}(t)=\dot{t} \mathbf{t}_{e}(t)
$$




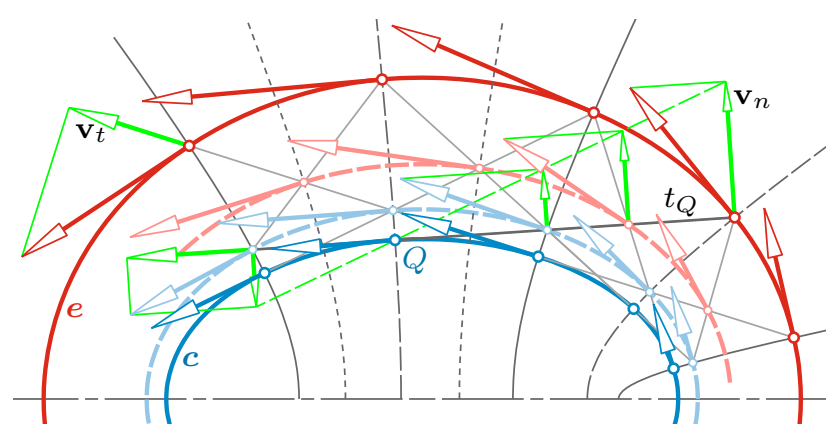

Fig. 5 The infinitesimal motion assigns to each point of the Poncelet grid a velocity vector such that on each tangent $t_{Q}$ to the caustic $c$ the points remain aligned

We can extend this to all confocal ellipses of the caustic. Then the assignment of a velocity vector $\mathbf{v}(t)$ to each point $P=\left(a_{e} \cos t, b_{e} \sin t\right)$ with $a_{e}^{2}-b_{e}^{2}=a_{c}^{2}-b_{c}^{2}$ defines an instant motion of the plane, where

$$
\dot{t}=\frac{\mathrm{d} t}{\mathrm{~d} u}=\left\|\mathbf{t}_{c}(t)\right\|=\sqrt{-k_{h}(t)}=\sqrt{a_{c}^{2} \sin ^{2} t+b_{c}^{2} \cos ^{2} t} .
$$

We prove below, that this instant motion is compatible with the billiard and the associated Poncelet grid. This means in particular, that the velocities in (3.5) are also valid for the induced movement of the grid points $S_{i}^{(j)}$ along $e^{(j)}$.

Figure 5 shows a portion of the Poncelet grid and the velocity vectors of a couple of points, each represented by a scaled arrow. As indicated, for any point $Q \in c$ all points on the tangent $t_{Q}$ have velocity vectors $\mathbf{v}$ where the respective normal components $\left\|\mathbf{v}_{n}\right\|$ are proportional to the distances to $Q$. On the other hand, all points on any confocal hyperbola share the tangential component $\left\|\mathbf{v}_{t}\right\|$ in accordance with (3.5).

Theorem 4.1 Let the billiard $P_{1} P_{2} \ldots$ with the ellipse $c$ as caustic be moving along the circumscribed ellipse e. Then the motion is the action of a one-parameter Lie group $\Gamma$. Each transformation $\gamma(u) \in \Gamma$ preserves the confocal ellipses and permutes the confocal hyperbolas as well as the tangents to $c$.

(1) If $\left(a_{c}, b_{c}\right)$ are the semiaxes of the caustic $c$ with the tangent vectors $\mathbf{t}_{c}(t)=$ $\left(-a_{c} \sin t, b_{c} \cos t\right)$, then for all confocal ellipses $e$ with semiaxes $\left(a_{e}, b_{e}\right)$ the $\Gamma$ generating instant motion is defined, up to a scalar, by the vector field

$$
(x, y)=\left(a_{e} \cos t, b_{e} \sin t\right) \mapsto\left\|\mathbf{t}_{c}\right\| \mathbf{t}_{e}=\sqrt{-k_{h}(t)}\left(-\frac{a_{e} y}{b_{e}}, \frac{b_{e} x}{a_{e}}\right)
$$

with $a_{e}^{2}-b_{e}^{2}=a_{c}^{2}-b_{c}^{2}=d^{2}$ and $a_{e}^{2}-a_{c}^{2} \geqslant 0$.

(2) If we parametrize the quadrant $x, y>0$ by elliptic coordinates as $\mathbf{X}\left(k_{e}, k_{h}\right)$, then the vector field can be expressed as

$$
\mathbf{X}\left(k_{e}, k_{h}\right) \mapsto-2 \sqrt{k_{h}\left(a_{c}^{2}+k_{h}\right)\left(b_{c}^{2}+k_{h}\right)} \frac{\partial \mathbf{X}}{\partial k_{h}} .
$$


Proof (1) The first derivative $\dot{t}$ in (4.2) is independent of the choice of the ellipse $e$. Therefore $\gamma(u)$ permutes the confocal hyperbolas. On the other hand, the representation $\mathbf{v}=\left\|\mathbf{t}_{c}\right\| \mathbf{t}_{e}$ reveals that all confocal ellipses remain fixed. Furthermore, we verify that the position of any point $P$ on the tangent $t_{Q}$ to $c$ at $Q$ (see Fig. 5) is preserved under the infinitesimal motion:

Given $P=\left(a_{e} \cos t, b_{e} \sin t\right) \in e$ and $Q=\left(a_{c} \cos t^{\prime}, b_{c} \sin t^{\prime}\right)$, the point $P$ lies on $t_{Q}$ if and only if

$$
b_{c} a_{e} \cos t^{\prime} \cos t+a_{c} b_{e} \sin t^{\prime} \sin t=a_{c} b_{c} .
$$

This is preserved under the infinitesimal motion if differentiation by $u$ based on (4.2) yields an identity, namely

$$
\begin{aligned}
\left\|\mathbf{t}_{c}\left(t^{\prime}\right)\right\| & \left(-b_{c} a_{e} \sin t^{\prime} \cos t+a_{c} b_{e} \cos t^{\prime} \sin t\right) \\
& =-\left\|\mathbf{t}_{c}(t)\right\|\left(-b_{c} a_{e} \cos t^{\prime} \sin t+a_{c} b_{e} \sin t^{\prime} \cos t\right) .
\end{aligned}
$$

In order to verify this, we square both sides and substitute from the squared equation (4.5) the mixed term $2 a_{c} b_{c} a_{e} b_{e} \sin t^{\prime} \cos t^{\prime} \sin t \cos t$. After some computations, this yields for both sides

$$
d^{2}\left(\sin ^{2} t-\sin ^{2} t^{\prime}\right)\left(a_{c}^{2} b_{e}^{2} \sin ^{2} t^{\prime} \sin ^{2} t+b_{c}^{2} a_{e}^{2} \cos ^{2} t^{\prime} \cos ^{2} t-a_{c}^{2} b_{c}^{2}\right) .
$$

The velocity analysis in (3.5) for the particular ellipse $e$ confirms, that also the signs of both sides in (4.6) are equal.

(2) From (2.3) it follows for $(x, y)=\mathbf{X}\left(k_{e}, k_{h}\right)$ that

$$
2 x \frac{\partial x}{\partial k_{h}}=\frac{a_{c}^{2}+k_{e}}{d^{2}}, \quad 2 y \frac{\partial y}{\partial k_{h}}=-\frac{b_{c}^{2}+k_{e}}{d^{2}}
$$

and therefore

$$
\mathbf{X}_{k_{h}}=\frac{\partial \mathbf{X}}{\partial k_{h}}=\frac{1}{2 d^{2}}\left(\frac{a_{c}^{2}+k_{e}}{x},-\frac{b_{c}^{2}+k_{e}}{y}\right)=\frac{-1}{2 d^{2} \sin t \cos t} \mathbf{t}_{e} .
$$

This implies by (2.4) and (2.6)

$$
\left\|\mathbf{t}_{c}\right\| \mathbf{t}_{e}=\lambda \mathbf{X}_{k_{h}} \text { with } \lambda=-2 a_{h} b_{h} \sqrt{-k_{h}}=-2 \sqrt{k_{h}\left(a_{c}^{2}+k_{h}\right)\left(b_{c}^{2}+k_{h}\right)}
$$

which confirms the claim in (4.4).

The vector field (4.3) defines a canonical parameter $u$ for the one-parameter Lie group $\Gamma$, i.e., for transformations $\gamma(u) \in \Gamma$ it holds $\gamma\left(u_{2}\right) \circ \gamma\left(u_{1}\right)=\gamma\left(u_{1}+u_{2}\right)$. At the same time, $u$ provides canonical coordinates ${ }^{1}$ on each confocal ellipse: If the

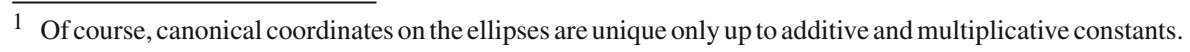


coordinates of $P_{2}$ and $P_{1}$ differ by $2 \Delta u$, then

$$
\gamma(2 \Delta u): P_{i} \mapsto P_{i+1}, Q_{i} \mapsto Q_{i+1}, S_{i}^{(j)} \mapsto S_{i+1}^{(j)} \text { for all } i \in\{1,2, \ldots\}
$$

According to (4.1), the velocity $v(t)$ of any point $Q=\left(a_{c} \cos t, b_{c} \sin t\right)$ on the caustic $c$ is given as

$$
v(t)=\left\|\mathbf{t}_{c}(t)\right\|^{2}=a_{c}^{2}+b_{c}^{2}-\overline{O Q}^{2},
$$

where $\overline{O Q}$ denotes the distance of $Q$ to the center $O$.

On the other hand, from (4.2) it follows that $\dot{t}=\left\|t_{c}(t)\right\|$. This implies in combination with the well-known formula $\kappa_{c}(t)=a_{c} b_{c} /\left\|\mathbf{t}_{c}(t)\right\|^{3}$ for the curvature of $c$ at $Q$ (see $\left[9\right.$, p.79]) that the arc length $s_{c}$ of the caustic $c$ satisfies

$$
\dot{s}_{c}=\frac{\mathrm{d} s_{c}}{\mathrm{~d} u}=\left\|\mathbf{t}_{c}(t)\right\| \dot{t}=\left\|\mathbf{t}_{c}(t)\right\|^{2}=\left(\frac{a_{c} b_{c}}{\kappa_{c}(t)}\right)^{2 / 3} .
$$

Herewith we confirm for the Euclidean plane Glutsyuk's formula for a Poritzky string length in $[10,(1.1)]$. From (2.4) there follows another expression.

Corollary 4.2 In terms of elliptic coordinates $\left(k_{e}, k_{h}\right)$ connected with the family of confocal conics of the caustic $c$, the arc length $s_{c}$ of $c$ and a Poritzky string length $u$ are related by

$$
\frac{\mathrm{d} s_{c}}{\mathrm{~d} u}=-k_{h}
$$

In order to express the action of the transformation $\gamma(u) \in \Gamma$ on an initial point $\left(a_{e} \cos t, b_{e} \sin t\right)$, we integrate (4.2)

$$
\dot{t}=\frac{\mathrm{d} t}{\mathrm{~d} u}=\sqrt{a_{c}^{2} \sin ^{2} t+\left(a_{c}^{2}-d^{2}\right) \cos ^{2} t}=a_{c} \sqrt{1-m^{2} \cos ^{2} t}
$$

with $m:=d / a_{c}<1$ as numerical eccentricity of the caustic $c$. The substitution

$$
\varphi:=t-\frac{\pi}{2}
$$

results in

$$
\frac{\mathrm{d} \varphi}{\sqrt{1-m^{2} \sin ^{2} \varphi}}=a_{c} \mathrm{~d} u
$$

The initial condition $\varphi=0$ for $u=0$ yields the unique solution

$$
a_{c} u(\varphi)=F(\varphi, m)=\int_{0}^{\varphi} \frac{\mathrm{d} \varphi}{\sqrt{1-m^{2} \sin ^{2} \varphi}}
$$


with $F(\varphi, m)$ as the elliptic integral of the first kind with the modulus $m$. The equation (4.7) shows the canonical coordinate $u$ in terms of $\varphi$ with the quarter period

$$
K:=a_{c} u\left(\frac{\pi}{2}\right)=\int_{0}^{\pi / 2} \frac{\mathrm{d} \varphi}{\sqrt{1-m^{2} \sin ^{2} \varphi}} .
$$

For the sake of simplicity, we introduce a new canonical coordinate by

$$
\tilde{u}(\varphi):=a_{c} u(\varphi)
$$

The inverse function of $\tilde{u}=F(\varphi, m)$, namely the Jacobian amplitude $\varphi=\operatorname{am}(\tilde{u})$ leads to the Jacobian elliptic functions, the elliptic sine

$$
\operatorname{sn} \tilde{u}=\sin (\operatorname{am}(\tilde{u}))=\sin \varphi=-\cos t
$$

with $\operatorname{sn}(-\tilde{u})=-\operatorname{sn} \tilde{u}$, the elliptic cosine

$$
\operatorname{cn} \tilde{u}=\cos (\operatorname{am}(\tilde{u}))=\cos \varphi=\sin t
$$

with $\operatorname{cn}(-\tilde{u})=\operatorname{cn} \tilde{u}$, and the delta amplitude

$$
\operatorname{dn} \tilde{u}=\sqrt{1-m^{2} \operatorname{sn}^{2} \tilde{u}}
$$

with $\operatorname{dn}(-\tilde{u})=\operatorname{dn} \tilde{u}$ as the third elliptic base function [12]. Moreover, for $k \in \mathbb{Z}$,

$$
\begin{aligned}
& \operatorname{sn}(\tilde{u}+2 k K)=(-1)^{k} \operatorname{sn} \tilde{u}, \\
& \operatorname{cn}(\tilde{u}+2 k K)=(-1)^{k} \operatorname{cn} \tilde{u}, \\
& \operatorname{dn}(\tilde{u}+2 k K)=(-1)^{k} \operatorname{dn} \tilde{u} .
\end{aligned}
$$

This gives rise to the canonical parametrization of the ellipse $e$ with semiaxes $\left(a_{e}, b_{e}\right)$ as

$$
\left(-a_{e} \operatorname{sn} \tilde{u}, b_{e} \operatorname{cn} \tilde{u}\right) \text { for } 0 \leqslant \tilde{u}<4 K=4 \tilde{u}\left(\frac{\pi}{2}\right) .
$$

As an alternative, we can proceed with elliptic coordinates. From (4.4) and

$$
\frac{\mathrm{d} \mathbf{X}}{\mathrm{d} u}=\dot{k}_{e} \frac{\partial \mathbf{X}}{\partial k_{e}}+\dot{k}_{h} \frac{\partial \mathbf{X}}{\partial k_{h}}=-2 \sqrt{k_{h}\left(a_{c}^{2}+k_{h}\right)\left(b_{c}^{2}+k_{h}\right)} \frac{\partial \mathbf{X}}{\partial k_{h}}
$$

it follows that for the orbits of the Lie group $\dot{k}_{e}=0$ and

$$
\dot{k}_{h}=-2 \sqrt{k_{h}\left(a_{c}^{2}+k_{h}\right)\left(b_{c}^{2}+k_{h}\right)} \text {. }
$$




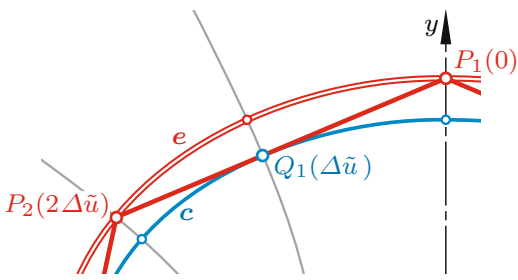

Fig. 6 Dependence between the minor semiaxes $b_{e}, b_{c}$ and the interval $\Delta \tilde{u}$

As expected, the orbits are confocal ellipses. Integration of

$$
\frac{\mathrm{d} k_{h}}{\sqrt{k_{h}\left(a_{c}^{2}+k_{h}\right)\left(b_{c}^{2}+k_{h}\right)}}=-2 \mathrm{~d} u
$$

gives again an elliptic integral, this time in the so-called Riemannian form.

Theorem 4.3 (1) Let $c$ be the ellipse $c$ with semiaxes $\left(a_{c}, b_{c}\right)$ and linear eccentricity $d=\sqrt{a_{c}^{2}-b_{c}^{2}}$. Then for all confocal ellipses $e$ with semiaxes $\left(a_{e}, b_{e}\right)$, the inscribed billiards with the caustic $c$ can be canonically parametrized using the Jacobian elliptic functions to the modulus $m=d / a_{c}$ (=numerical eccentricity of c) as

$$
\left(-a_{e} \operatorname{sn} \tilde{u}, b_{e} \operatorname{cn} \tilde{u}\right) \text {. }
$$

If $b_{c}=b_{e} \operatorname{cn}(\Delta \tilde{u})$, then the vertices of the billiards in $e$ with caustic $c$ have the canonical parameters $\tilde{u}=\left(\tilde{u}_{1}+2 k \Delta \tilde{u}\right)$ for $k \in \mathbb{Z}$ and any given initial $\tilde{u}_{1}$.

(2) Conversely, we obtain an ellipse e for which the billiards with caustic $c$ are $N$ periodic with turning number $\tau$, where $\operatorname{gcd}(N, \tau)=1$, by the choice

$$
\Delta \tilde{u}=\frac{2 \tau K}{N}
$$

with $K$ as the complete elliptic integral of the first kind to the modulus $m$, provided that

$$
a_{e}=\frac{a_{c} \operatorname{dn}(\Delta \tilde{u})}{\operatorname{cn}(\Delta \tilde{u})} \quad \text { and } \quad b_{e}=\frac{b_{c}}{\operatorname{cn}(\Delta \tilde{u})} .
$$

Remark 4.4 It needs to be noted that already in 1828 Jacobi presented an analogue condition for the periodicity, when he treated a projectively equivalent case with $e$ and $c$ as nested circles (see [14, p. 388]).

Proof If the first vertex $P_{1} \in e$ of the billiard is chosen on the positive $y$-axis, i.e., with canonical parameter $\tilde{u}=0$ (see Fig. 6), then the first contact point $Q_{1}$ has the parameter $\Delta \tilde{u}$, and the tangent to $c$ at $Q_{1}$ passes through $P_{1}=\left(0, b_{e}\right)$. Hence, the points $P_{1}$ and $Q_{1}$ are conjugate w.r.t. $c$, which means by (4.5) that the product of the respective $y$ coordinates $b_{e}$ and $b_{c} \mathrm{cn}(\Delta \tilde{u})$ equals $b_{c}^{2}$. Moreover, from $\operatorname{dn}^{2}(\Delta \tilde{u})=1-m^{2} \operatorname{sn}^{2}(\Delta \tilde{u})$ and $\operatorname{sn}^{2}(\Delta \tilde{u})+\operatorname{cn}^{2}(\Delta \tilde{u})=1$ it follows that $\operatorname{dn}(\Delta \tilde{u})=a_{e} \operatorname{cn}(\Delta \tilde{u}) / a_{c}$. 


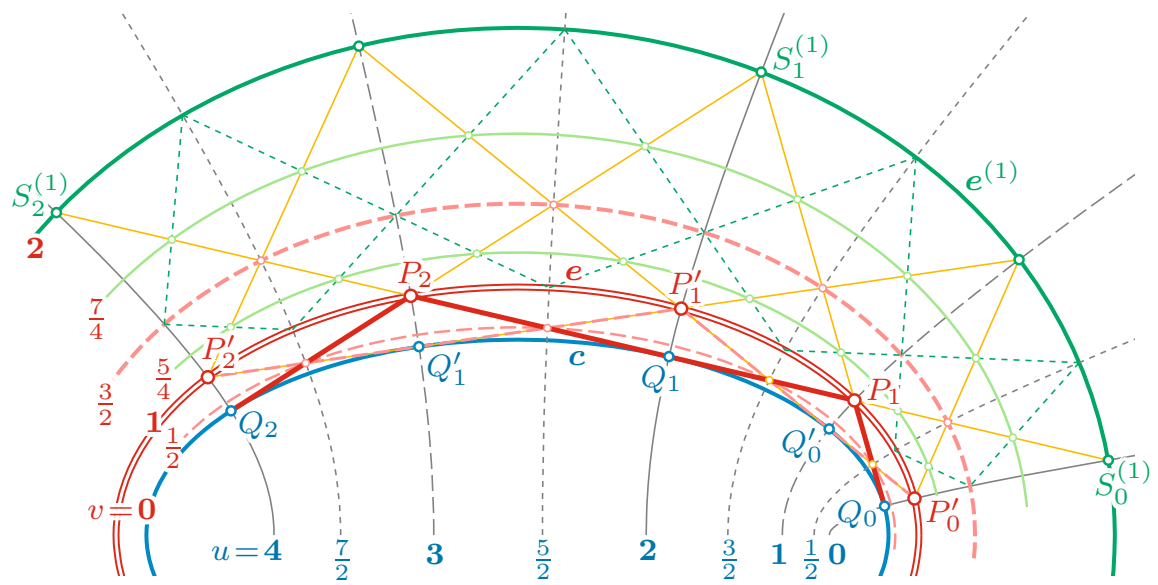

Fig. 7 Canonical coordinates $u$ (blue) for the confocal hyperbolas and $v$ (red) for the confocal ellipses exterior to the caustic $c$ such that $u \pm v=$ const. represent the tangents of the caustic

Corollary 4.5 If in the ellipse $e$ with semiaxes $\left(a_{e}, b_{e}\right)$ the billiard with caustic $c$ is $N$-periodic with turning number $\tau=1$ and $\Delta \tilde{u}=2 K / N$, then the associated Poncelet grid contains the ellipses $e^{(1)}, e^{(2)}, \ldots, e^{(k)}, k=[(N-3) / 2]$, with respective semiaxes

$$
\begin{gathered}
a_{e \mid 1}=\frac{a_{c} \operatorname{dn}(2 \Delta \tilde{u})}{\operatorname{cn}(2 \Delta \tilde{u})}, b_{e \mid 1}=\frac{b_{c}}{\operatorname{cn}(2 \Delta \tilde{u})}, \quad a_{e \mid 2}=\frac{a_{c} \operatorname{dn}(3 \Delta \tilde{u})}{\operatorname{cn}(3 \Delta \tilde{u})}, b_{e \mid 2}=\frac{b_{c}}{\operatorname{cn}(3 \Delta \tilde{u})}, \\
\ldots, a_{e \mid k-1}=\frac{a_{c} \operatorname{dn}(k \Delta \tilde{u})}{\operatorname{cn}(k \Delta \tilde{u})}, \quad b_{e \mid k-1}=\frac{b_{c}}{\operatorname{cn}(k \Delta \tilde{u})} .
\end{gathered}
$$

As illustrated in Fig. 7, the Poncelet grid provides an intuitive approach to canonical coordinates on the confocal ellipses $c$ of $e$ : Let us assign the coordinate 0 to $Q_{0}$ and 2 to $Q_{1}$. Then the confocal hyperbolas through vertices of the grid and billiards inscribed in confocal ellipses allow an iterated bisection and extension to further contact points.

Corollary 4.5 reveals that $\Delta \tilde{u}$ serves as a canonical coordinate for confocal ellipses in the exterior of $c$. If $\Delta \tilde{u}$ corresponds by (4.9) to the ellipse $e$ with semiaxes $\left(a_{e}, b_{e}\right)$, then $2 \Delta \tilde{u}$ is the shift for the billiards in $e$ with caustic $c$. If these billiards have the turning number 1 , then increasing the shift by $\Delta \tilde{u}$ means to increase the turning number of the billiard in a confocal ellipse by 1 , while the caustic $c$ remains fixed (Fig. 7). The billiard $P_{1} P_{2} \ldots$ and its conjugate $P_{1}^{\prime} P_{2}^{\prime} \ldots$ in $e$ (cf. [20, Section 3.2]) intersect each other along the ellipse with the canonical coordinate $\Delta \tilde{u} / 2$. Note that for $N$-periodic billiards the ellipses $e^{(j)}$ and $e^{(N-2-j)}$ coincide while the corresponding $\Delta \tilde{u}$ 's differ in their signs. For even $N$, the points $S_{i}^{(N / 2-1)}$ are at infinity, and the line at infinity as a limit of a confocal ellipse corresponds to $\Delta \tilde{u}=K$.

The following formulas express the elliptic coordinates $\left(k_{e}, k_{h}\right)$ of the point $P=$ $\left(-a_{e} \operatorname{sn} \tilde{u}, b_{e} \operatorname{cn} \tilde{u}\right)$ of $e$ in terms of the canonical coordinate $\tilde{u}$ on $e$ and the shift $\Delta \tilde{u}$ corresponding to $e$. 


$$
k_{e}=k_{e}(\Delta \tilde{u})=\frac{a_{c}^{2} \operatorname{sn}^{2} \Delta \tilde{u}}{\mathrm{cn}^{2} \Delta \tilde{u}}\left(1-m^{2}\right), \quad k_{h}=k_{h}(\tilde{u})=-a_{c}^{2} \operatorname{dn}^{2} \tilde{u} .
$$

This follows from

$$
k_{e}=a_{e}^{2}-a_{c}^{2}=a_{c}^{2} \frac{\mathrm{dn}^{2} \Delta \tilde{u}-\mathrm{cn}^{2} \Delta \tilde{u}}{\mathrm{cn}^{2} \Delta \tilde{u}}=a_{c}^{2}\left(1-m^{2}\right) \frac{\mathrm{sn}^{2} \Delta \tilde{u}}{\mathrm{cn}^{2} \Delta \tilde{u}}
$$

and

$$
k_{h}=-a_{c}^{2} \mathrm{cn}^{2} \tilde{u}-b_{c}^{2} \operatorname{sn}^{2} \tilde{u}=-a_{c}^{2}+d^{2} \operatorname{sn}^{2} \tilde{u}=-a_{c}^{2}+m^{2} a_{c}^{2} \operatorname{sn}^{2} \tilde{u} .
$$

Note that $k_{h}=k_{h}(\tilde{u})$ is a solution of (4.8).

In [13], an unordered pair of coordinates $(r, s)$ is proposed for each point $P$ in the exterior of $c$, namely with $r$ and $s$ as canonical coordinates of the tangency points for the tangent lines from $P$ to $c$ (see also [18, p. 358]). This means for $P=\left(-a_{e}\right.$ sn $\tilde{u}, b_{e}$ cn $\left.\tilde{u}\right)$ that

$$
r=\tilde{u}-\Delta \tilde{u}, \quad s=\tilde{u}+\Delta \tilde{u},
$$

where $\Delta \tilde{u}$ corresponds to $e$ according to (4.9). If the sum $\tilde{u}+\Delta \tilde{u}$ remains constant or the difference $\tilde{u}-\Delta \tilde{u}$, then the corresponding point $P$ runs along a tangent of the caustic $c$ (compare with [3, Proposition 8.3]).

The coordinates $\tilde{u}=(r+s) / 2$ and $\tilde{v}:=\Delta \tilde{u}=(s-r) / 2$ give rise to a new representation of a mapping from a zig-zag polygon to a billiard, which was already studied in [7, Figure 13]. Below we extend it to a mapping from a square grid to a Poncelet grid. ${ }^{2}$

Theorem 4.6 Referring to the notation in Theorem 4.3, the injective mapping

$$
\begin{gathered}
\mathbf{Y}: U \times V \rightarrow \mathbb{R}^{2}, \quad(\tilde{u}, \tilde{v}) \mapsto\left(-a_{c} \frac{\operatorname{sn} \tilde{u} \operatorname{dn} \tilde{v}}{\operatorname{cn} \tilde{v}}, b_{c} \frac{\operatorname{cn} \tilde{u}}{\operatorname{cn} \tilde{v}}\right) \\
\quad \text { for } U:=\{\tilde{u} \mid 0 \leqslant \tilde{u}<4 K\}, \quad V:=\{\tilde{v} \mid 0 \leqslant \tilde{u}<K\}
\end{gathered}
$$

parametrizes the exterior of the caustic $c$ with semiaxes $\left(a_{c}, b_{c}\right)$ in such a way, that the lines $\tilde{u}=$ const. are branches of confocal hyperbolas; $\tilde{v}=$ const. are confocal ellipses and $\tilde{u} \pm \tilde{v}=$ const. tangents of $c$.

The domain of the mapping $\mathbf{Y}$ can be extended to $\mathbb{R}^{2}$ and satisfies

$$
\mathbf{Y}((\tilde{u}+4 K), \tilde{v})=\mathbf{Y}(\tilde{u},(\tilde{v}+2 K))=\mathbf{Y}(\tilde{u},-\tilde{v})=\mathbf{Y}(\tilde{u}, \tilde{v})
$$

and therefore $\mathbf{Y}(\tilde{u},(K+\tilde{v}))=\mathbf{Y}(\tilde{u},(K-\tilde{v}))($ Fig. 8). The Lie group $\Gamma$ mentioned in Theorem 4.1 is the $\mathbf{Y}$-transform of the group of translations along the $\tilde{u}$-axis.

\footnotetext{
2 A similar mapping and its generalization to 3-space was used in [3] from the viewpoint of discrete confocal coordinate systems.
} 


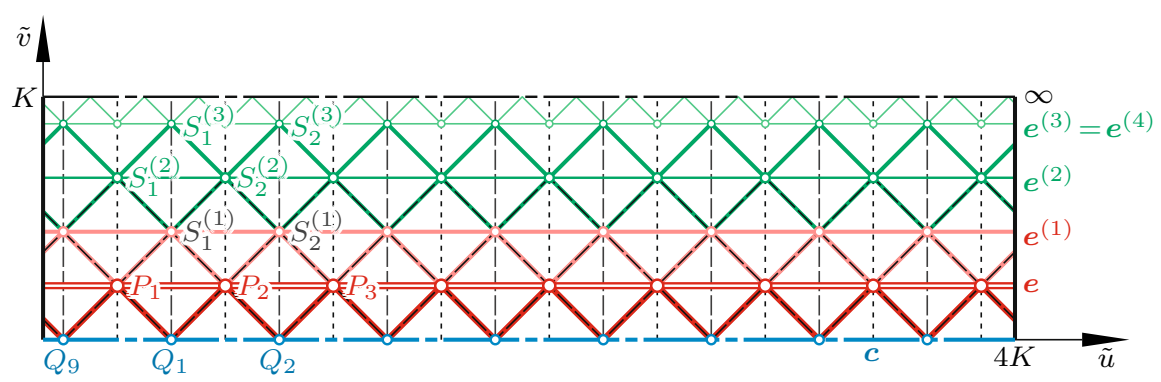

Fig. 8 The injective mapping $\mathbf{Y}$ sends the square grid of points $Q_{i}, P_{i}$ and $S_{i}^{(j)}, i=1, \ldots, 9, j=1, \ldots, 3$, to the vertices and the diagonals to the confocal conics of the Poncelet grid depicted in Fig. 2

\section{More about invariants of periodic billiards}

In this section we study how the infinitesimal motion induced by the vector field in (4.3) affects distances and angles at the billiard. As before, the dot means differentiation by the canonical parameter $u$.

Lemma 5.1 Let $P_{1} P_{2} \ldots$ be a billiard in the ellipse $e$ with $Q_{1}, Q_{2}, \ldots$ as contact points with its caustic, the ellipse c. If $P_{i}=\left(a_{e} \cos t_{i}, b_{e} \sin t_{i}\right)$ and $Q_{i}=$ $\left(a_{c} \cos t_{i}^{\prime}, b_{c} \sin t_{i}^{\prime}\right)$, then the distances $r_{i}:=\overline{Q_{i-1} P_{i}}$ and $l_{i}:=\overline{P_{i} Q_{i}}$ satisfy

$$
r_{i}=\frac{\left\|\mathbf{t}_{c}\left(t_{i-1}^{\prime}\right)\right\|\left\|\mathbf{t}_{c}\left(t_{i}\right)\right\| \sqrt{k_{e}}}{a_{c} b_{c}}, \quad l_{i}=\frac{\left\|\mathbf{t}_{c}\left(t_{i}\right)\right\|\left\|\mathbf{t}_{c}\left(t_{i}^{\prime}\right)\right\| \sqrt{k_{e}}}{a_{c} b_{c}} .
$$

The velocity vectors in (4.3) induce for $P_{i} P_{i+1}$ the angular velocity

$$
\omega_{i}=\frac{a_{c} b_{c}}{\left\|\mathbf{t}_{c}\left(t_{i}^{\prime}\right)\right\|}=\frac{a_{c} b_{c}}{\sqrt{-k_{h}\left(t_{i}^{\prime}\right)}}
$$

Proof Referring to Fig. 9, if the tangent $\left[Q_{1}, P_{2}\right]$ rolls on $c$, then the vertex $P_{2}$ receives the velocity vector $\mathbf{v}_{n_{2}}$ satisfying (3.5), while the point of contact $Q_{1}$ moves with the velocity $v_{c}\left(t_{1}^{\prime}\right)$ along $c$. We can express this velocity in terms of the radius of curvature $\rho_{c}\left(t_{1}^{\prime}\right)$ of $c$ as

$$
v_{c}\left(t_{1}^{\prime}\right)=\omega_{1} \rho_{c}\left(t_{1}^{\prime}\right)
$$

where $\rho_{c}(t)=\left\|\mathbf{t}_{e}(t)\right\|^{3} / a_{c} b_{c}$ by [9, p. 79]. On the other hand, from $\mathbf{v}_{c}=\dot{t} \mathbf{t}_{c}$ and (4.2) it follows that $v_{c}\left(t_{1}^{\prime}\right)=\left\|\mathbf{t}_{c}\left(t_{1}^{\prime}\right)\right\|^{2}$. This yields in accordance with Corollary 4.2 ,

$$
\omega_{1}=\frac{v_{c}\left(t_{1}^{\prime}\right)}{\rho_{c}\left(t_{1}^{\prime}\right)}=\frac{\left\|\mathbf{t}_{c}\left(t_{1}^{\prime}\right)\right\|^{2} a_{c} b_{c}}{\left\|\mathbf{t}_{c}\left(t_{1}^{\prime}\right)\right\|^{3}}=\frac{a_{c} b_{c}}{\left\|\mathbf{t}_{c}\left(t_{1}^{\prime}\right)\right\|}=\frac{a_{c} b_{c}}{\sqrt{-k_{h}\left(t_{1}^{\prime}\right)}}
$$




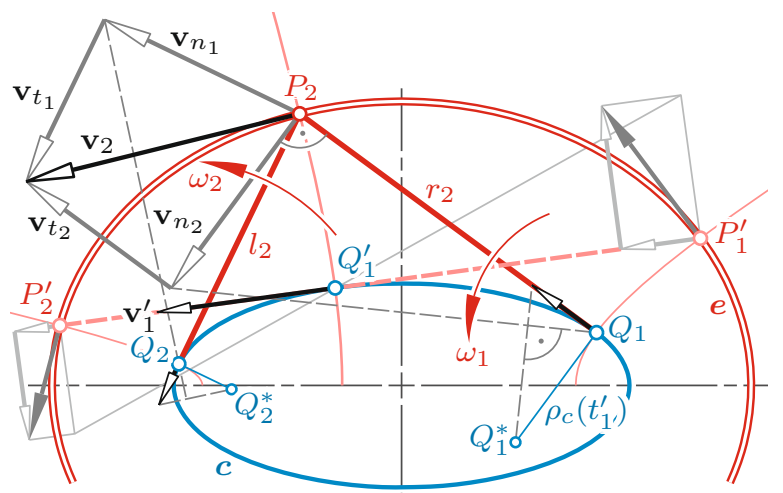

Fig. 9 Velocity $\mathbf{v}_{2}$ of the vertex $P_{2}, \mathbf{v}_{1}^{\prime}$ of the affine image $Q_{1}^{\prime}$ with $\left\|\mathbf{v}_{1}^{\prime}\right\|=\left\|\mathbf{v}_{n_{1}}\right\|=\left\|\mathbf{v}_{n_{2}}\right\|$, of the contact points $Q_{i}$ (with $Q_{i}^{*}$ as respective centers of curvature) for $i=1,2$, and of vertices $P_{i}^{\prime}$ of the conjugate billiard

by (2.4). Thus, we obtain for the velocity $v_{n \mid 2}$ of $P_{2}$ by (3.5),

$$
r_{2} \omega_{1}=v_{n \mid 2}=\left\|\mathbf{t}_{c}\left(t_{2}\right)\right\| \sqrt{k_{e}}, \quad \text { hence } \quad r_{2}=\frac{\left\|\mathbf{t}_{c}\left(t_{2}\right)\right\| \sqrt{k_{e}}}{a_{c} b_{c}}\left\|\mathbf{t}_{c}\left(t_{1}^{\prime}\right)\right\| .
$$

Similarly, it follows from $l_{2} \omega_{2}=v_{n \mid 2}$ the stated expression for the distance $l_{2}$. Note that $t_{1}, t_{1}^{\prime}, t_{2}, t_{2}^{\prime}, t_{3}, \ldots$ is the sequence of consecutive parameters of the points $P_{1}, Q_{1}, P_{2}, Q_{2}, P_{3}, \ldots$ The formulas for $r_{i}$ and $l_{i}$ as well as Ivory's theorem reveal that the same distances appear as $l_{i-1}^{\prime}$ and $r_{i}^{\prime}$ at the conjugate billiard.

The angular velocity of the tangent to $e$ at $P_{2}$ equals the arithmetic mean $\left(\omega_{1}+\omega_{2}\right) / 2$ (Fig. 9). On the other hand, it is defined by the radius of curvature $\rho_{e}$ of $e$ at $P_{2}$ and the velocity $v_{2}$ by (3.5), since

$$
v_{2}=\rho_{e}\left(t_{2}\right) \frac{\omega_{1}+\omega_{2}}{2}
$$

This means by Lemma 5.1,

$$
\left\|\mathbf{t}_{c}\left(t_{2}\right)\right\|\left\|\mathbf{t}_{e}\left(t_{2}\right)\right\|=\frac{\left\|\mathbf{t}_{e}\left(t_{2}\right)\right\|^{3}}{a_{e} b_{e}} \frac{a_{c} b_{c}}{2}\left(\frac{1}{\left\|\mathbf{t}_{c}\left(t_{1}^{\prime}\right)\right\|}+\frac{1}{\left\|\mathbf{t}_{c}\left(t_{2}^{\prime}\right)\right\|}\right)
$$

and results by (2.5) in

$$
\frac{1}{\left\|\mathbf{t}_{c}\left(t_{1}^{\prime}\right)\right\|}+\frac{1}{\left\|\mathbf{t}_{c}\left(t_{2}^{\prime}\right)\right\|}=\frac{2 a_{e} b_{e}}{a_{c} b_{c}} \frac{\left\|\mathbf{t}_{c}\left(t_{2}\right)\right\|}{\left\|\mathbf{t}_{e}\left(t_{2}\right)\right\|^{2}}=\frac{a_{e} b_{e}}{a_{c} b_{c} \sqrt{k_{e}}} \sin \theta_{2} .
$$

Theorem 5.2 The exterior angles $\theta_{i}$ of an $N$-periodic billiard in an ellipse and with an ellipse as caustic satisfy for even $N$ 


$$
\sum_{i=1}^{N}(-1)^{i} \sin \theta_{i}=0 \text { and for } N \equiv 0(\bmod 4) \sum_{i=1}^{N / 2}(-1)^{i} \sin \theta_{i}=0
$$

Proof By virtue of [20, Corollary 4.2], periodic billiards with even $N=2 n$ are centrally symmetric, which implies $\theta_{i}=\theta_{i+n}$. For $N \equiv 2(\bmod 4)$ the sum from 1 to $N$ must vanish since $(-1)^{i}=-(-1)^{(i+n)}$.

In the remaining case $N \equiv 0(\bmod 4)$ it follows from $(5.1)$ that

$$
\sin \theta_{i}=\frac{a_{c} b_{c} \sqrt{k_{e}}}{a_{e} b_{e}}\left(\frac{1}{\left\|\mathbf{t}_{c}\left(t_{i-1}^{\prime}\right)\right\|}+\frac{1}{\left\|\mathbf{t}_{c}\left(t_{i}^{\prime}\right)\right\|}\right)
$$

and further

$$
\begin{aligned}
\sum_{i=1}^{N / 2} \sin \theta_{i}= & \frac{a_{c} b_{c} \sqrt{k_{e}}}{a_{e} b_{e}} \\
& \cdot\left(\frac{1}{\left\|\mathbf{t}_{c}\left(t_{N}^{\prime}\right)\right\|}+\frac{1}{\left\|\mathbf{t}_{c}\left(t_{1}^{\prime}\right)\right\|}-\frac{1}{\left\|\mathbf{t}_{c}\left(t_{1}^{\prime}\right)\right\|}-\frac{1}{\left\|\mathbf{t}_{c}\left(t_{2}^{\prime}\right)\right\|}+-\cdots-\frac{1}{\left\|\mathbf{t}_{c}\left(t_{n}^{\prime}\right)\right\|}\right) .
\end{aligned}
$$

This sum vanishes, since $\mathbf{t}_{c}\left(t_{n}^{\prime}\right)=-\mathbf{t}_{c}\left(t_{N}^{\prime}\right)$, due to the odd turning number $\tau$ because of $\operatorname{gcd}(N, \tau)=1$.

At the same token, from

$$
\dot{\theta}_{i}=\omega_{i}-\omega_{i-1}=a_{c} b_{c}\left(\frac{1}{\left\|\mathbf{t}_{c}\left(t_{i}^{\prime}\right)\right\|}-\frac{1}{\left\|\mathbf{t}_{c}\left(t_{i-1}^{\prime}\right)\right\|}\right)
$$

and (5.1) it follows that

$$
\frac{\mathrm{d}}{\mathrm{d} u} \cos \theta_{i}=-\dot{\theta}_{i} \sin \theta_{i}=\frac{a_{c}^{2} b_{c}^{2} \sqrt{k_{e}}}{a_{e} b_{e}}\left(\frac{1}{\left\|\mathbf{t}_{c}\left(t_{i-1}^{\prime}\right)\right\|^{2}}-\frac{1}{\left\|\mathbf{t}_{c}\left(t_{i}^{\prime}\right)\right\|^{2}}\right) .
$$

This shows that $\frac{\mathrm{d}}{\mathrm{d} u}\left(\sum_{1}^{N} \cos \theta_{i}\right)$ vanishes and, therefore, $\sum_{1}^{N} \cos \theta_{i}$ is invariant against billiard motions, which was first proved in [1].

For the variation of side lengths it follows that

$$
\begin{aligned}
\frac{\mathrm{d}}{\mathrm{d} u} \overline{P_{i} P_{i+1}} & =v_{t \mid i+1}-v_{t \mid i} \\
& =\left\|\mathbf{t}_{c}\left(t_{i+1}\right)\right\|^{2}-\left\|\mathbf{t}_{c}\left(t_{i}\right)\right\|^{2}=d^{2}\left(\sin ^{2} t_{i+1}-\sin ^{2} t_{i}\right) .
\end{aligned}
$$

The vanishing sum over all $i$ confirms again the constant perimeter. We recall that already in [2] some proofs for invariants were based on differentiation.

Finally we concentrate on the effects showing up when the vertex $P_{i}$ traverses a quarter of the full period along a periodic billiard. 
Lemma 5.3 As before, let $t_{1}, t_{1}^{\prime}, t_{2}, t_{2}^{\prime}, \ldots, t_{N}^{\prime}$ be the sequence of parameters of an $N$-periodic billiard in an ellipse $e$ with an ellipse $c$ as caustic. Then the following equations hold for even $N$ and $n \in \mathbb{Z}$ :

$$
\left.\begin{array}{rl}
\text { if } N=4 n \text {, then }\left\|\mathbf{t}_{c}\left(t_{i}\right)\right\|\left\|\mathbf{t}_{c}\left(t_{i+n}\right)\right\| & =\sqrt{k_{h}\left(t_{i}\right) k_{h}\left(t_{i+n}\right)} \\
\text { if } N=4 n+2, \text { then }\left\|\mathbf{t}_{c}\left(t_{i}\right)\right\|\left\|\mathbf{t}_{c}\left(t_{i+n}^{\prime}\right)\right\| & =\sqrt{k_{h}\left(t_{i}\right) k_{h}\left(t_{i+n}^{\prime}\right)}
\end{array}\right\}=a_{c} b_{c},
$$

and the same after the parameter shift $t_{i} \mapsto t_{i}^{\prime}$ and $t_{i}^{\prime} \mapsto t_{i+1}$.

Proof Based on the canonical parametrization by $\tilde{u}$, a quarter of the period $4 K$ corresponds to a shift by $K$. In the case $N=4 n$ this shift effects $t_{i} \mapsto t_{i+n}$ and $t_{i}^{\prime} \mapsto t_{i+n}^{\prime}$. If $N=4 n+2$, then $t_{i} \mapsto t_{i+n}^{\prime}$ and $t_{i}^{\prime} \mapsto t_{i+n+1}$.

According to (4.10), $k_{h}=-a_{c}^{2} \operatorname{dn}^{2} \tilde{u}$ and by (2.4), $\left\|\mathbf{t}_{c}(t)\right\|=\sqrt{-k_{h}(t)}=a_{c} \operatorname{dn} \tilde{u}$. The identity

$$
\operatorname{dn}(\tilde{u}+K)=\frac{\sqrt{1-m^{2}}}{\operatorname{dn}(\tilde{u})}
$$

implies

$$
\operatorname{dn}(\tilde{u}) \cdot \operatorname{dn}(\tilde{u}+K)=\frac{b_{c}}{a_{c}}, \text { hence } \sqrt{k_{h}(\tilde{u}) \cdot k_{h}(\tilde{u}+K)}=a_{c} b_{c} .
$$

This confirms the claim.

Theorem 5.4 If the billiard $P_{1} P_{2} \ldots P_{N}$ in the ellipse $e$ is $N$-periodic and contacts the ellipse $c$ as caustic at $Q_{1} Q_{2} \ldots Q_{N}$, then for even $N$ the distances $r_{i}=\overline{Q_{i-1} P_{i}}$ and $l_{i}=\overline{P_{i} Q_{i}}$ satisfy

$$
\left.\begin{array}{l}
\text { for } \quad N=4 n: \quad r_{i} \cdot r_{i+n}=l_{i} \cdot l_{i+n} \\
\text { for } \quad N=4 n+2: r_{i} \cdot l_{i+n}=l_{i} \cdot r_{i+n+1}
\end{array}\right\}=k_{e} .
$$

Proof From the expressions for $r_{i}$ and $l_{i}$ in Lemma 5.1, by virtue of Lemma 5.3, for $N=4 n$,

$$
r_{i} \cdot r_{i+n}=\frac{k_{e}}{a_{c}^{2} b_{c}^{2}}\left\|\mathbf{t}_{c}\left(t_{i-1}^{\prime}\right)\right\|\left\|\mathbf{t}_{c}\left(t_{i}\right)\right\|\left\|\mathbf{t}_{c}\left(t_{i+n-1}^{\prime}\right)\right\|\left\|\mathbf{t}_{c}\left(t_{i+n}\right)\right\|=k_{e}
$$

and the same result holds for $l_{i} \cdot l_{i+n}$. In the case $N=4 n+2$ we obtain similarly

$$
r_{i} \cdot l_{i+n}=l_{i} \cdot r_{i+n+1}=k_{e},
$$

as stated.

The following corollary is an immediate consequence of Theorem 5.4. 
Corollary 5.5 Let $s_{i}=\overline{P_{i} P_{i+1}}=l_{i}+r_{i+1}$ for $i=1, \ldots, N$ be the side lengths of an $N$-periodic billiard with even $N$ and $s_{i}^{\prime}=\overline{P_{i}^{\prime} P_{i+1}^{\prime}}=r_{i+1}+l_{i+1}$ that of the conjugate billiard. Then,

$$
\begin{array}{r}
\text { for } N=4 n: \frac{s_{i+n}}{s_{i}}=\frac{l_{i+n}}{r_{i+1}}=\frac{r_{i+n+1}}{l_{i}}, \\
\text { for } N=4 n+2: \frac{s_{i+n}}{s_{i-1}^{\prime}}=\frac{l_{i+n}}{l_{i}}=\frac{r_{i+n+1}}{r_{i}} .
\end{array}
$$

Finally we prove the invariance of k117 in [17, Table 2].

Theorem 5.6 Referring to the notation in Lemma 5.1, for even $N$ the products

$$
r_{1} r_{2} \ldots r_{N}=l_{1} l_{2} \ldots l_{N}=k_{e}^{N / 2}
$$

are invariant against billiard motions. For $N \equiv 0(\bmod 4)$ this is already true for the products

$$
r_{1} r_{2} \ldots r_{N / 2}=l_{1} l_{2} \ldots l_{N / 2}=k_{e}^{N / 4}
$$

Proof For $N \equiv 0(\bmod 4)$ the statements are a direct consequence of Theorem 5.4 and the central symmetry of the billiard which exchanges $r_{i}$ with $r_{i+N / 2}$ and $l_{i}$ with $l_{i+N / 2}$. In the remaining case $N=2 n+2$ we note that by (3.2), $R(u):=r_{1} r_{2} \ldots r_{N}=$ $l_{1} l_{2} \ldots l_{N}$. Hence, by virtue of Theorem 5.4 ,

$$
R^{2}(u)=\prod_{i=1}^{N}\left(r_{i} l_{i+n}\right)=k_{e}^{N},
$$

which yields the stated result.

Funding Open access funding provided by TU Wien (TUW).

Open Access This article is licensed under a Creative Commons Attribution 4.0 International License, which permits use, sharing, adaptation, distribution and reproduction in any medium or format, as long as you give appropriate credit to the original author(s) and the source, provide a link to the Creative Commons licence, and indicate if changes were made. The images or other third party material in this article are included in the article's Creative Commons licence, unless indicated otherwise in a credit line to the material. If material is not included in the article's Creative Commons licence and your intended use is not permitted by statutory regulation or exceeds the permitted use, you will need to obtain permission directly from the copyright holder. To view a copy of this licence, visit http://creativecommons.org/licenses/by/4.0/.

\section{References}

1. Akopyan, A., Schwartz, R., Tabachnikov, S.: Billiards in ellipses revisited. Eur. J. Math. https://doi. org/10.1007/s40879-020-00426-9

2. Bialy, M., Tabachnikov, S.: Dan Reznik's identities and more. Eur. J. Math. https://doi.org/10.1007/ s40879-020-00428-7 
3. Bobenko, A.I., Schief, W.K., Suris, Yu..B., Techter, J.: On a discretization of confocal quadrics. A geometric approach to general parametrizations. Int. Math. Res. Not. IMRN 2020(24), 10180-10230 (2020)

4. Chavez-Caliz, A.C.: More about areas and centers of Poncelet polygons. Arnold Math. J. 7(1), 91-106 (2021)

5. Dragović, V., Radnović, M.: Integrable billiards and quadrics. Russian Math. Surv. 65(2), 319-379 (2010)

6. Dragović, V., Radnović, M.: Poncelet Porisms and Beyond. Frontiers in Mathematics. Birkhäuser, Basel (2011)

7. Dragović, V., Radnović, M.: Pseudo-integrable billiards and arithmetic dynamics. J. Mod. Dyn. 8(1), 109-132 (2014)

8. Duistermaat, J..J.: Discrete Integrable Systems. QRT Maps and Elliptic Surfaces. Springer Monographs in Mathematics, vol. 304. Springer, New York (2010)

9. Glaeser, G., Stachel, H., Odehnal, B.: The Universe of Conics. Springer Spectrum, Berlin (2016)

10. Glutsyuk, A.: On curves with Poritsky property (2019). arXiv:1901.01881 [math.DS]

11. Glutsyuk, A., Izmestiev, I., Tabachnikov, S.: Four equivalent properties of integrable billiards. Israel J. Math. 241(2), 693-719 (2021)

12. Hoppe, R.: Elliptische Integrale und Funktionen nach Jacobi. http://www.dfcgen.de/wpapers/elliptic. pdf (2004-2015), accessed May 2021

13. Izmestiev, I., Tabachnikov, S.: Ivory's theorem revisited. J. Integrable Syst. 2(1), xyx006 (2017)

14. Jacobi, C.G.J.: lieber die Anwendung der elliptischen Transcendenten auf ein bekanntes Problem der Elementargeometrie. J. Reine Angew. Math. 3, 376-389 (1828)

15. King, J.L.: Three problems in search of a measure. Amer. Math. Monthly 101(7), 609-628 (1994)

16. Poritsky, H.: The billiard ball problem on a table with a convex boundary-an illustrative dynamical problem. Ann. Math. 51, 446-470 (1950)

17. Reznik, D., Garcia, R., Koiller, J.: Eighty new invariants of $N$-periodics in the elliptic billiard (2020). arXiv:2004.12497v11 [math.DS]

18. Stachel, H.: Recalling Ivory's theorem. FME Trans. 47(2), 355-359 (2019)

19. Stachel, H.: Isometric billiards in ellipses and focal billiards in ellipsoids. J. Geom. Graph. 25(1), 97-118 (2021)

20. Stachel, H.: The geometry of billiards in ellipses and their Poncelet grids. J. Geom. 112(3), 30 (2021)

21. Tabachnikov, S.: Geometry and Billiards. Student Mathematical Library, vol. 30. American Mathematical Society, Providence (2005)

Publisher's Note Springer Nature remains neutral with regard to jurisdictional claims in published maps and institutional affiliations. 